\title{
Testing the QGSJET01 and QGSJETII-04 models with the help of atmospheric muons
}

\author{
Leonid G. Dedenko ${ }^{1,2, a}$, Anton V. Lukyashin ${ }^{3,4, b}$, Tatiana M. Roganova ${ }^{2, c}$, and Galina F. Fedorova ${ }^{2, d}$ \\ ${ }^{1}$ Faculty of Physics M.V. Lomonosov Moscow State University, Leninskie Gory, 119991 Moscow, Russia \\ ${ }^{2}$ Skobeltsyn Institute of Nuclear Physics, Lomonosov Moscow State University, 119234 Moscow, Russia \\ ${ }^{3}$ State Scientific Center Institute for Theoretical and Experimental Physics, National Research Center "Kurchatov Institute", 117218 \\ Moscow, Russia \\ ${ }^{4}$ National Research Nuclear University (MEPhI) Moscow Engineering Physics Institute, 115409 Moscow, Russia
}

\begin{abstract}
More accurate original calculations of the atmospheric vertical muon energy spectra at energies $10^{2}-10^{5} \mathrm{GeV}$ have been carried out in terms of the QGSJET01 and QGSJETII-04 models. The GaisserHonda approximations of the measured energy spectra of primary protons, helium and nitrogen nuclei have been used. The CORSIKA package has been used to simulate cascades in the standard atmosphere induced by different primary particles with various fixed energies $E$. Statistics of simulated cascades for secondary particles with energies $(0.01-1) \cdot E$ was increased up to $10^{6}$. It has been shown that predictions of the QGSJET01 and QGSJETII-04 models for these muon fluxes are below the data of the classical experiments L3 + Cosmic, MACRO and LVD by factors of $\sim 1.7-2$ at energies above $10^{2} \mathrm{GeV}$. It has been concluded that these tested models underestimate the production of the most energetic secondary particles, namely, $\pi$-mesons and $K$-mesons, in interactions of primary protons and other primary nuclei with nuclei in the atmosphere by the same factors.
\end{abstract}

\section{Introduction}

Extensive air showers (EAS) are used as a tool to understand the origin and composition of cosmic rays, their possible sources and the transport of cosmic particles in various magnetic fields on their way to the Earth at very high energies. All features of the energy spectrum, arrival directions and composition of the primary cosmic particles should be determined through an analysis of the EAS data. These data as some signals in the surface and underground detectors are usually interpreted in terms of various models of hadronic interactions [1-8]. Such interpretation may be not obligatory correct. As an example, the energy of showers calculated in terms of the QGSJETII-03 [2] model with the help of the surface detectors signals at the Telescope Array (TA) [9] happened to be 1.27 times lager than such energy estimated with the help of the fluorescence light. To ensure that results of such an interpretation are as accurate as possible these models should be thoroughly tested. Usually these models are tested with the help of the accelerator data at small values $(\sim 0)$ of the pseudorapidity, $\eta$, where most of secondary particles are produced [10-12]. However, calculations have shown that the maximal energy flow carried by secondary particles occurs at much larger values $(\sim 8-10)$ of the pseudorapidity [13]. Let us also note that the longitudinal development of EAS depends strongly on the rate of the projectile particle energy fragmentation.

\footnotetext{
a e-mail: ddn@dec1.sinp.msu.ru

b e-mail: lukyashin.anton@physics.msu.ru

c e-mail: rogatm@yandex.ru

d e-mail: fdr@dec1.sinp.msu.ru
}

So, it is of primary importance to verify the production of the most energetic secondary particles simulated in terms of these models. The atmospheric muon flux also depends strongly on such a production. So, it is a valuable suggestion to test various models of hadronic interactions by comparing model predictions of these muon fluxes with data. There are many beautiful data to be compared with model predictions. We select the classical experiments L3+Cosmic [14], MACRO [15] and LVD [16] and elaborate the smooth approximation of these muon data in the energy interval of $10^{2}-10^{5} \mathrm{GeV}$. To make model predictions of the muon flux we have suggested to simulate EAS induced by primary protons, helium and nitrogen nuclei with different fixed energies in the atmosphere with the help of the CORSIKA package [17] and calculate the muon energy spectrum in each individual shower. Results of these simulations for every type of primary particles multiplied by intensities of these primary particles should be integrated on the energy of these primary particles. Thus, we also need some expressions for the energy spectra of various primary particles. Indeed, there are results of many measurements (e.g. ATIC-2 [18], CREAM [19], RUNJOB [20], AMS02 [21,22], PAMELA [23]) of the fluxes of the primary cosmic nuclei. We will use the Gaisser-Honda approximations [24] for energy spectra of the various primary nuclei. Thus, with the help of any interaction models [1-8] and the package CORSIKA [17] and data on fluxes of the primary cosmic nuclei [18-23] or any approximations of the data, one can predict the energy spectra of atmospheric vertical high energy muons at sea level. These predictions can be compared with data observed by the L3+Cosmic [14], MACRO [15] 
and LVD [16] collaborations at energies above $100 \mathrm{GeV}$. Finally, some conclusions can be drawn about the validity of various models.

In fact, some low energies have been tested with the FLUKA [25] package in such a way. We are sorry that our testing of some models in [26-28] are not correct. We do apologize for our mistake in input data for the atmosphere!

In this paper models QGSJET01 [1] and QGSJETII04 [3] have been tested. A comparison of muon data observed in references [14-16] with the results of simulations allows us to draw a conclusion about the most energetic secondary particle production described by these models.

\section{Method}

The hadronic interaction models QGSJET01 and QGSJETII-04 have been tested with the help of the atmospheric muon data. To estimate the energy spectra $D\left(E_{\mu}\right)$ of atmospheric vertical muons in the energy range $10^{2}-10^{5} \mathrm{GeV}$ we need to know the energy spectra $d I_{p} / d E, d I_{H e} / d E$ and $d I_{N} / d E$ of the primary protons, $\mathrm{He}$ and $\mathrm{N}$ nuclei within the energy interval $10^{2}-10^{7}$ $\mathrm{GeV}$ and the energy spectra of vertical muons $S_{p}\left(E_{\mu}, E\right)$, $S_{H e}\left(E_{\mu}, E\right)$ and $S_{N}\left(E_{\mu}, E\right)$ calculated from the primary protons, helium and nitrogen nuclei with various fixed energies, $E$, in terms of the QGSJET01 and QGSJETII04 hadronic interaction models in the same energy range of $10^{2}-10^{5} \mathrm{GeV}$. For a comparison we need data on the vertical muon flux. The smooth approximation of the atmospheric muon data observed by the collaborations L3+Cosmic, MACRO and LVD had been used for comparison with results of simulations.

Functions $S_{p}\left(E_{\mu}, E\right), S_{H e}\left(E_{\mu}, E\right), S_{N}\left(E_{\mu}, E\right)$ are the differential energy spectra of muons in showers induced by primary protons, helium and nitrogen nuclei with fixed values of energy, $E$. These spectra were calculated for 24 values of the energy $E$ of the primary protons. The energy distributions of muons induced by primary helium and nitrogen nuclei $S_{H e}\left(E_{\mu}, E\right), S_{N}\left(E_{\mu}, E\right)$ have been also calculated as a combined distributions of protons based on the hypothesis of superposition [29]. As results coincide with simulations in terms of superposition, we will use this hypothesis for all nuclei.

The energy spectra of the primary particles are important ingredients of simulations. As the energy per nucleon is of importance, only the energy spectra of the primary protons, $\mathrm{He}$ and $\mathrm{N}$ nuclei should be taken into account. We had used approximations (1) for $\left(d I_{p} / d E\right)_{G H}$, $\left(d I_{H e} / d E\right)_{G H}$ and $\left(d I_{N} / d E\right)_{G H}$ suggested by Gaisser and Honda $(\mathrm{GH})$.

We have taken into account a possible change of primary spectrum above the "knee". At energies above $E_{1}=3 \cdot 10^{6} \mathrm{GeV}$ for primary protons and above $E_{2}=6 \cdot 10^{6} \mathrm{GeV}$ for primary $\mathrm{He}$ and $\mathrm{N}$ nuclei we used modified GH approximations (2) of the energy spectra of primary particles. The values of parameters for the Gaisser-Honda approximation are listed in Table 1. The approximation parameters: $\alpha, b$ and $c$ are dimensionless units. Parameter $K$ has dimensions $\left[1 /\left(G e V \cdot m^{2} \cdot s \cdot s r\right)\right]$. $E_{k}$ is the kinetic energy per nucleon in $\mathrm{GeV}$ units.

$$
\frac{d N}{d E_{k}}=K \cdot\left(E_{k}+b \cdot \exp \left(-c \cdot \sqrt{E_{k}}\right)\right)^{-\alpha}
$$

Table 1. Parameters for the Gaisser-Honda approximation.

\begin{tabular}{llllll}
\hline Nuclei & $\mathrm{A}$ & $\alpha$ & $K$ & $b$ & $c$ \\
\hline $\mathrm{H}$ & 1 & 2,74 & 14900 & 2,15 & 0,21 \\
$\mathrm{He}$ & 4 & 2,64 & 600 & 1,25 & 0,14 \\
$\mathrm{~N}$ & 14 & 2,6 & 33,2 & 0,97 & 0,01 \\
\hline
\end{tabular}

Table 2. Parameters for simulations: proton energy $E$ and threshold energy $E_{t h}$ in $\mathrm{GeV}$ and statistics $N$.

\begin{tabular}{lll|lll}
\hline$E$ & $E_{\text {th }}$ & $N$ & $E$ & $E_{\text {th }}$ & $N$ \\
\hline 113,3 & 100 & $10^{6}$ & $3,162 \cdot 10^{4}$ & 100 & $10^{6}$ \\
177,8 & 100 & $10^{6}$ & $5,623 \cdot 10^{4}$ & 100 & $10^{6}$ \\
237,1 & 100 & $10^{6}$ & $1 \cdot 10^{5}$ & 100 & $10^{5}$ \\
316,2 & 100 & $10^{6}$ & $1,778 \cdot 10^{5}$ & 100 & $10^{5}$ \\
421,7 & 100 & $10^{6}$ & $3,162 \cdot 10^{5}$ & 100 & $10^{5}$ \\
562,3 & 100 & $10^{6}$ & $5,623 \cdot 10^{5}$ & 100 & $10^{5}$ \\
749,8 & 100 & $10^{6}$ & $1 \cdot 10^{6}$ & 100 & $10^{4}$ \\
$1 \cdot 10^{3}$ & 100 & $10^{6}$ & $1,778 \cdot 10^{6}$ & 100 & $10^{4}$ \\
$1,778 \cdot 10^{3}$ & 100 & $10^{6}$ & $3,162 \cdot 10^{6}$ & 100 & $10^{4}$ \\
$3,162 \cdot 10^{3}$ & 100 & $10^{6}$ & $5,623 \cdot 10^{6}$ & 100 & $10^{4}$ \\
$5,623 \cdot 10^{3}$ & 100 & $10^{6}$ & $1 \cdot 10^{7}$ & 100 & $10^{3}$ \\
$1 \cdot 10^{4}$ & 100 & $10^{6}$ & $1,778 \cdot 10^{7}$ & 100 & $10^{3}$ \\
$1,778 \cdot 10^{4}$ & 100 & $10^{6}$ & $3,162 \cdot 10^{7}$ & 100 & $10^{3}$ \\
\hline \hline $1 \cdot 10^{5}$ & $10^{3}$ & $10^{6}$ & $1 \cdot 10^{6}$ & $10^{4}$ & $10^{6}$ \\
$1,778 \cdot 10^{5}$ & $10^{3}$ & $10^{6}$ & $1,778 \cdot 10^{6}$ & $10^{4}$ & $10^{6}$ \\
$3,162 \cdot 10^{5}$ & $10^{3}$ & $10^{6}$ & $3,162 \cdot 10^{6}$ & $10^{4}$ & $10^{6}$ \\
$5,623 \cdot 10^{5}$ & $10^{3}$ & $10^{6}$ & $5,623 \cdot 10^{6}$ & $10^{4}$ & $10^{6}$ \\
\hline
\end{tabular}

$$
\frac{d N}{d E_{k}}=K \cdot\left(E_{k}+b \cdot \exp \left(-c \cdot \sqrt{E_{k}}\right)\right)^{-\alpha} \cdot \sqrt{\frac{E_{i}}{E_{k}}}
$$

The CORSIKA 7.4 package had been used to simulate the second important ingredients - the energy spectra $S_{p}\left(E_{\mu}, E\right), S_{H e}\left(E_{\mu}, E\right)$ and $S_{N}\left(E_{\mu}, E\right)$ of vertical muons in showers induced by primary protons, helium and nitrogen nuclei for various fixed energies, $E$, in terms of the QGSJET01 and QGSJETII-04 hadronic interaction models in the energy range of $10^{2}-10^{5} \mathrm{GeV}$ with statistics $10^{6}$ events for the most energetic muons. Table 2 shows fixed energies, $E$, of the primary protons and statistics of simulated showers.

The results of these calculations in the energy range $10^{2}-10^{7} \mathrm{GeV}$ were interpolated for 100 values of energies $E$ with equal intervals in decimal logarithmic scale. The energy interval $10^{2}-10^{5} \mathrm{GeV}$ of muons was divided into 60 equal bins also in a decimal logarithmic scale. So, the width of the bin was equal to $h=\lg \left(E_{\mu,(i+1)} / E_{\mu, i}\right)=0,05$. Let us note that average muon energies for the 1-st, 21-st and 41-st bins which we will use later are equal to $105.9,1.059 \cdot 10^{3}$ and $1.059 \cdot 10^{4} \mathrm{GeV}$ respectively. In fact simulations for $\mathrm{He}$ and $\mathrm{N}$ nuclei have been carried out only for energies $10^{4}$ and $10^{6} \mathrm{GeV}$ to test the hypothesis of superposition. As results of simulations for the primary nuclei showed a good agreement with this hypothesis we used this hypothesis to estimate the flux of the nucleons from the primary helium and nitrogen nuclei.

The energy spectra $D_{p}\left(E_{\mu}\right), D_{H e}\left(E_{\mu}\right)$ and $D_{N}\left(E_{\mu}\right)$ of muons for the primary protons, He and $\mathrm{N}$ nuclei are calculated as integrals of the products of functions $S_{p}\left(E_{\mu}, E\right)$, $S_{H e}\left(E_{\mu}, E\right)$ and $S_{N}\left(E_{\mu}, E\right)$ with corresponding intensities $d I_{p} / d E, d I_{H e} / d E$ and $d I_{N} / d E$ of the primary protons, on the energy, $E$, of primary particles. 


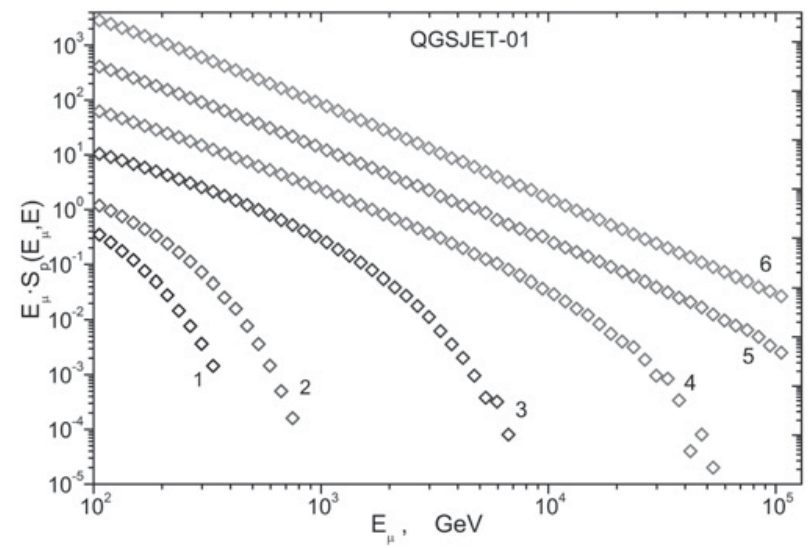

Figure 1. The energy spectra of muons in showers induced by primary protons with various fixed energies E (QGSJET01 model): $1-5 \cdot 10^{2} ; 2-10^{3} ; 3-10^{4} ; 4-10^{5} ; 5-10^{6} ; 6-10^{7} \mathrm{GeV}$.

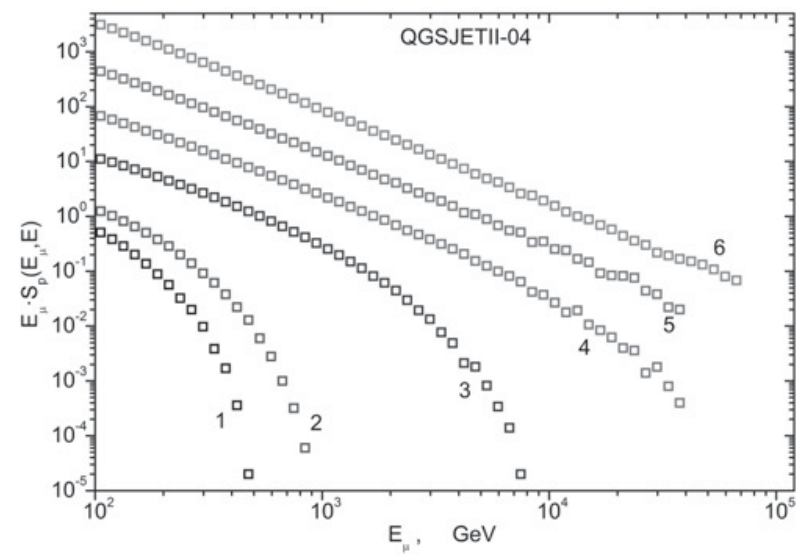

Figure 2. The energy spectra of muons in showers induced by primary protons with various fixed energies E (QGSJETII-04 model): $1-5 \cdot 10^{2} ; 2-10^{3} ; 3-10^{4} ; 4-10^{5} ; 5-10^{6} ; 6-10^{7} \mathrm{GeV}$.

$$
\begin{gathered}
D_{p}\left(E_{\mu}\right)=\int\left(\frac{d I_{p}}{d E}\right) \cdot S_{p}\left(E_{\mu}, E\right) \cdot d E \\
D_{H e}\left(E_{\mu}\right)=\int\left(\frac{d I_{H e}}{d E}\right) \cdot S_{H e}\left(E_{\mu}, E\right) \cdot d E \\
D_{N}\left(E_{\mu}\right)=\int\left(\frac{d I_{N}}{d E}\right) \cdot S_{N}\left(E_{\mu}, E\right) \cdot d E
\end{gathered}
$$

The resulting energy spectrum of atmospheric muons is the sum of partial energy spectra of muons produced by primary protons, helium and nitrogen nuclei

$$
D\left(E_{\mu}\right)=D_{p}\left(E_{\mu}\right)+D_{H e}\left(E_{\mu}\right)+D_{N}\left(E_{\mu}\right) .
$$

\section{Results of simulations}

The energy spectra $S_{p}\left(E_{\mu}, E\right)$ of the atmospheric vertical muons simulated for various fixed energies, $E$, of primary protons in terms of the QGSJET01 and QGSJETII-04 hadronic interaction models are shown in Figs. 1 and 2 respectively. It is seen that statistics of $\sim 10^{6}$ at the higher energy end of the spectra is not enough.
Table 3. Average number of muons with energies above the threshold $E_{t h}$ in showers induced by primary protons with energies $E$.

\begin{tabular}{lclll}
\hline \multicolumn{5}{c|}{$E=10^{5} \mathrm{GeV}$} \\
\hline Model & Paper & $E_{t h}$ & $100 \mathrm{GeV}$ & $1000 \mathrm{GeV}$ \\
\hline QGSJET01 & {$[30]$} & 21,0 & 0,605 \\
QGSJET01 & This work & 20,9 & 0,593 \\
QGSJETII-04 & This work & 22,4 & 0,613 \\
\hline \hline \multicolumn{4}{c}{$E=10^{6} \mathrm{GeV}$} \\
\hline Model & Paper $E_{t h}$ & $100 \mathrm{GeV}$ & $1000 \mathrm{GeV}$ \\
\hline QGSJET01 & {$[30]$} & 132,3 & 3,612 \\
QGSJET01 & This work & 132,8 & 3,490 \\
QGSJETII-04 & This work & 141,3 & 3,583 \\
\hline
\end{tabular}

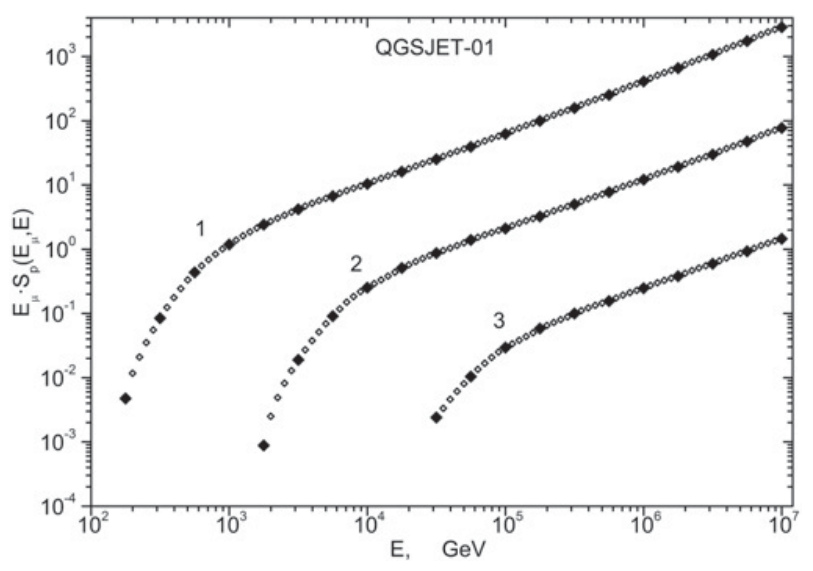

Figure 3. Dependence of $E_{\mu} \cdot S_{p}\left(E_{\mu}, E\right)$ on $E$ for 3 muon bins. $1-E_{\mu}=105,9 \mathrm{GeV} ; 2-E_{\mu}=1,059 \cdot 10^{3} \mathrm{GeV} ; E_{\mu}=1,059$. $10^{4} \mathrm{GeV}$. $\checkmark$-direct calculation, $\diamond$-interpolated results.

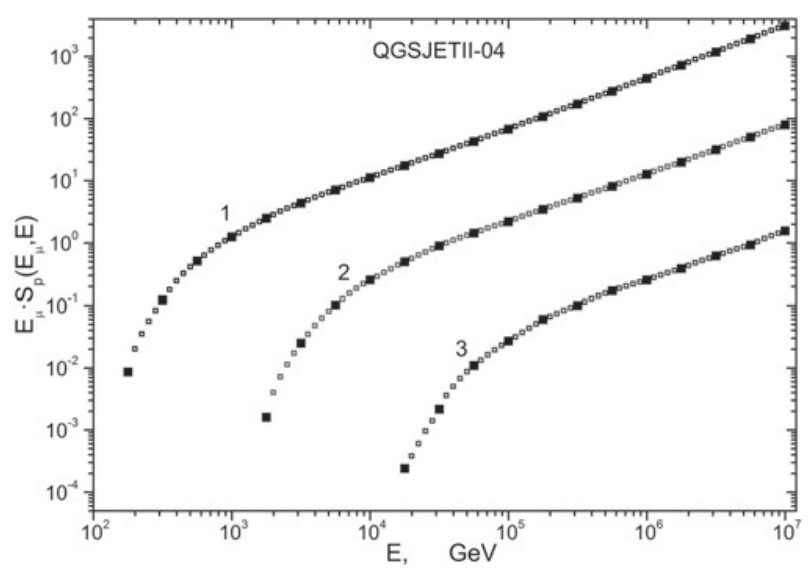

Figure 4. Dependence of $E_{\mu} \cdot S_{p}\left(E_{\mu}, E\right)$ on $E$ for 3 muon bins. $1-E_{\mu}=105,9 \mathrm{GeV} ; 2-E_{\mu}=1,059 \cdot 10^{3} \mathrm{GeV} ; E_{\mu}=1,059$. $10^{4} \mathrm{GeV}$. -direct calculation, $\square$-interpolated results.

Table 3 displays the total number of muons with energies above $10^{2}$ and $10^{3} \mathrm{GeV}$ in showers induced by primary protons with energies $10^{5}$ and $10^{6} \mathrm{GeV}$ estimated in terms of the QGSJET01 and QGSJETII-04 hadronic interaction models in our simulations and in reference [30]. A very reasonable agreement is seen.

Results of interpolation on energy, $E$, for the 1-st, 21-st and 41-st bins of muon energy spectra $S_{p}\left(E_{\mu}, E\right)$ as open symbols and direct results as solid symbols are displayed in Figs. 3 and 4 for the QGSJET01 and QGSJETII-04 models respectively. Good agreement is also seen. 


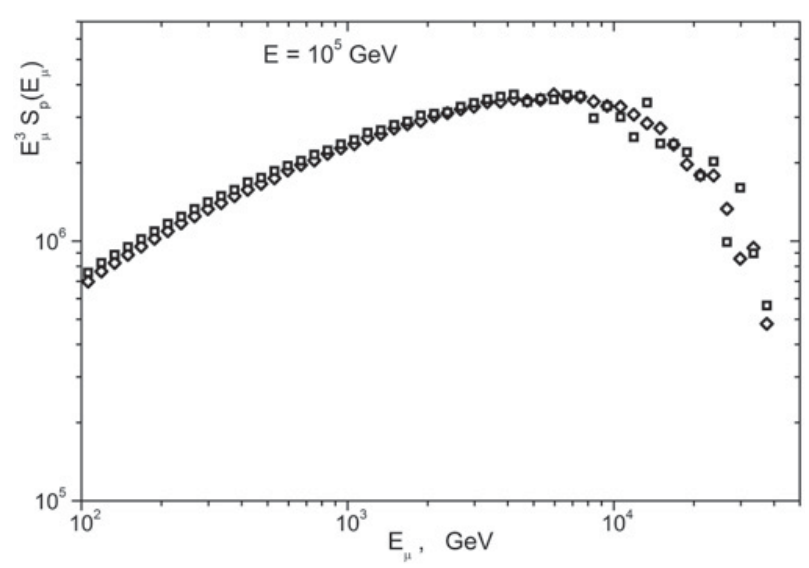

Figure 5. The energy spectra of muons in showers induced by primary protons with fixed energy $\mathrm{E}=10^{5} \mathrm{GeV}$. $\diamond$ - QGSJET01, $\square$ - QGSJETII-04.

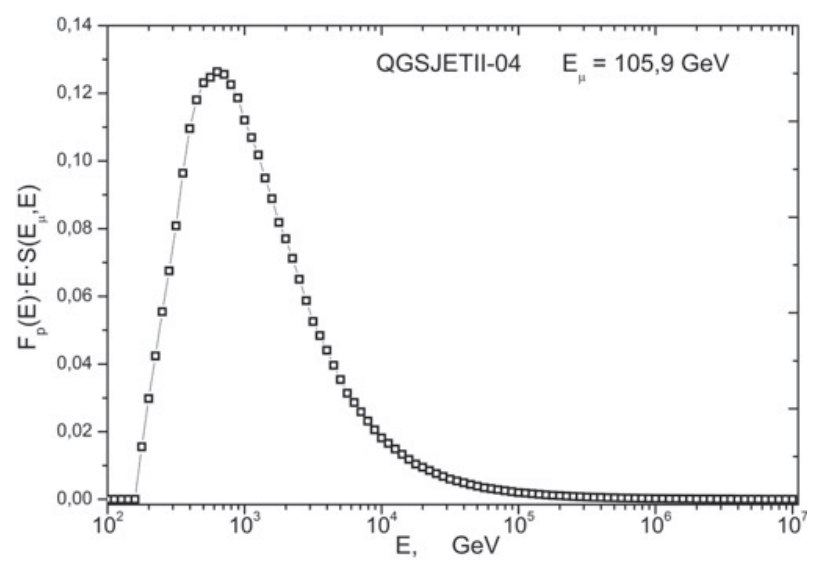

Figure 6. Contributions of the primary protons with various energies $E$ into the 1-st bin of the muon spectra.

The next Fig. 5 demonstrates a comparison of the muon energy spectra $S_{p}\left(E_{\mu}, E\right)$ calculated in terms of the QGSJET01 model as open diamonds and the QGSJETII04 model as solid squares for a fixed energy $E=10^{5} \mathrm{GeV}$ of the primary protons. The results for the QGSJETII-04 model are 10-15\% above the QGSJET01 model values.

Figures 6, 7 and 8 demonstrate distributions of the primary nucleon energy which contribute much to the 1 -st, 21-st and 41-st bins of muon energy spectra respectively for the QGSJETII-04 model. It is possible to note that nearly two orders of energy, $E$, are of importance for any fixed energy, $E_{\mu}$, of muons. The maximal contributions occur at energies $\sim 7 \cdot 10^{2} \mathrm{GeV}$ (1-st bin), $\sim 7 \cdot 10^{3} \mathrm{GeV}$ (21-st bin) and $\sim 6,4 \cdot 10^{4} \mathrm{GeV}$ (41-st bin). The results of comparison of calculations in terms of the QGSJET01 and QGSJETII-04 models spectra $D\left(E_{\mu}\right)$ with the smooth approximation of data [14-16] are illustrated in Fig. 9. It should be noted that at the above energies $\sim 100 \mathrm{GeV}$ both the simulated spectra and data are steepened. It is because the decay constant, $B$, for the charged mesons is equal to $B \sim 100 \mathrm{GeV}$ and the probability of decay for charged mesons with higher energies is decreasing.

It is seen that the calculated spectra are 2 times below the data for the case of the QGSJET01 model and 1.7 times below the data for the QGSJETII-04 model. Figure 10

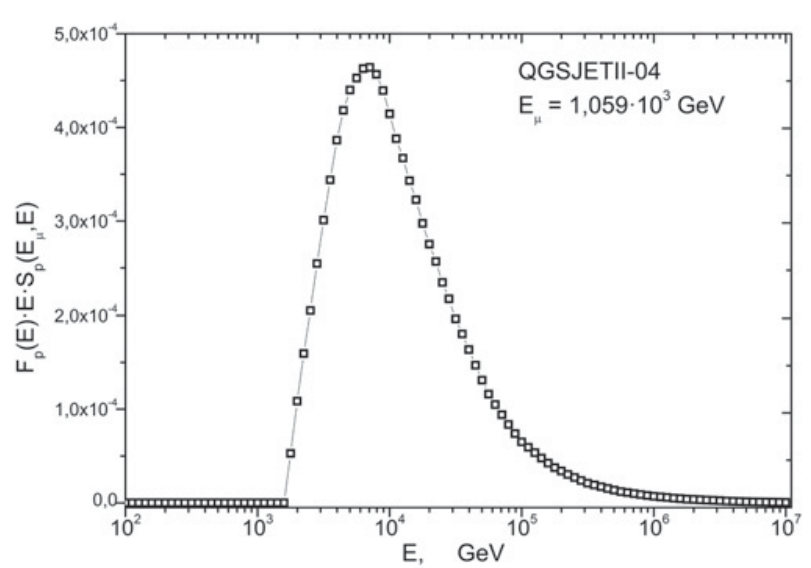

Figure 7. Contributions of the primary protons with various energies $E$ in to the 21 -st bin of the muon spectra.

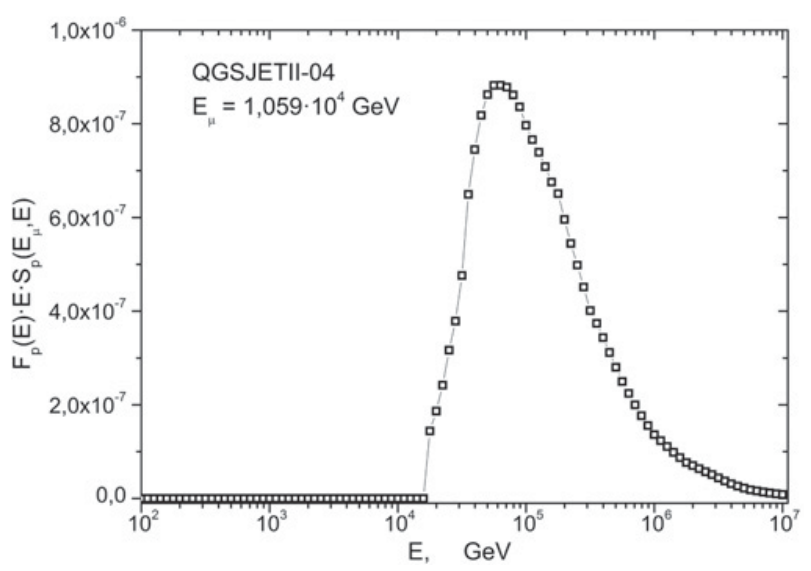

Figure 8. Contributions of primary protons with various energies $E$ in to the 41-st bin of the muon spectra.

shows ratios MC/DATA for the two models. For the case of the QGSJET01 model this ratio decrease from 55\% at $E=10^{2} \mathrm{GeV}$ to $50 \%$ at $E=10^{5} \mathrm{GeV}$ while in case of the QGSJETII-04 model this ratio decreases from 65\% to $55 \%$ for the same values of muon energy $E$. Figure 11 shows various contributions to the total muon energy spectrum $D\left(E_{\mu}\right)$ from the primary protons, $\mathrm{He}$ and $\mathrm{N}$ nuclei. These contributions are decreasing from 90 to $85 \%$ for protons and are increasing from 9 to $14 \%$ for $\mathrm{He}$ nuclei and from 0,5 to $1 \%$ for $\mathrm{N}$ nuclei. These fractions are not exactly reliable because the true fraction of nuclei is unknown.

\section{Conclusion}

Muons which contributes much to the muon energy spectra are produced in decays of the most energetic $\pi$-mesons and $K$-mesons generated in the first interactions of the primary particles with nuclei in the atmosphere. As the calculated vertical muon energy spectra for the QGSJET01 and QGSJETII-04 models are 1.7 times and 2 times respectively below the data we can conclude that the production of the most energetic $\pi$-mesons and $K$-mesons in these models is considerably suppressed. This suppression may induce smaller values of signals in the surface scintillation detectors and will result in larger values of the calculated energy estimates. So, the 


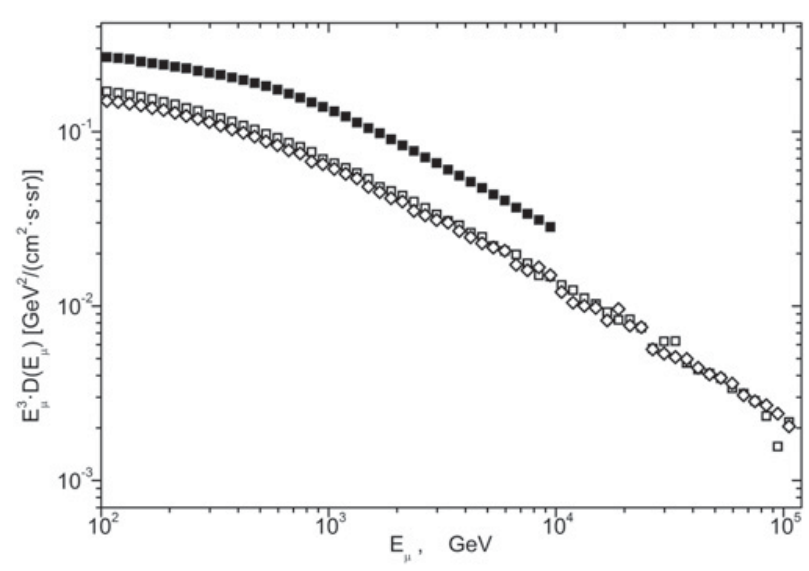

Figure 9. The energy spectra of near vertical muons. $\diamond$-QGSJET01, $\square$-QGSJETII-04, and (ם)-experimental data (L3+Cosmic, MACRO, LVD).

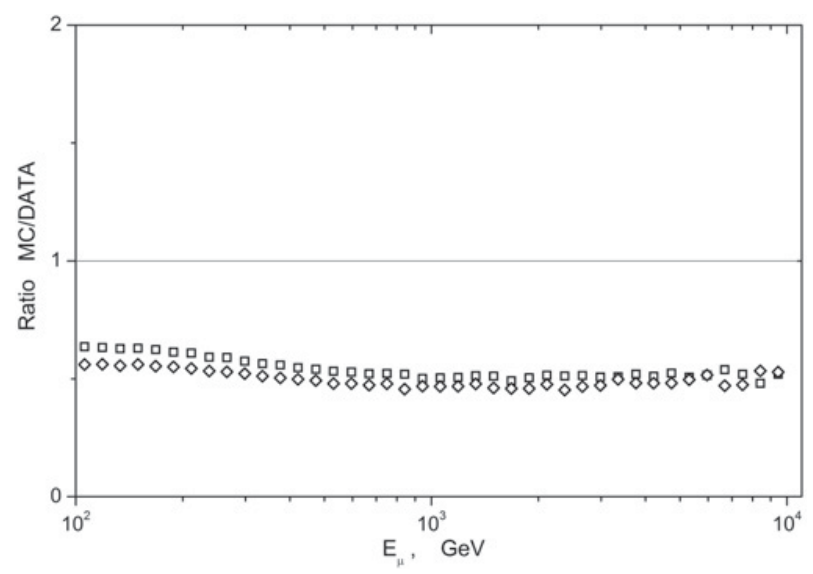

Figure 10. The ratio MC/DATA: $\diamond$-QGSJET01, $\square$-QGSJETII-04.

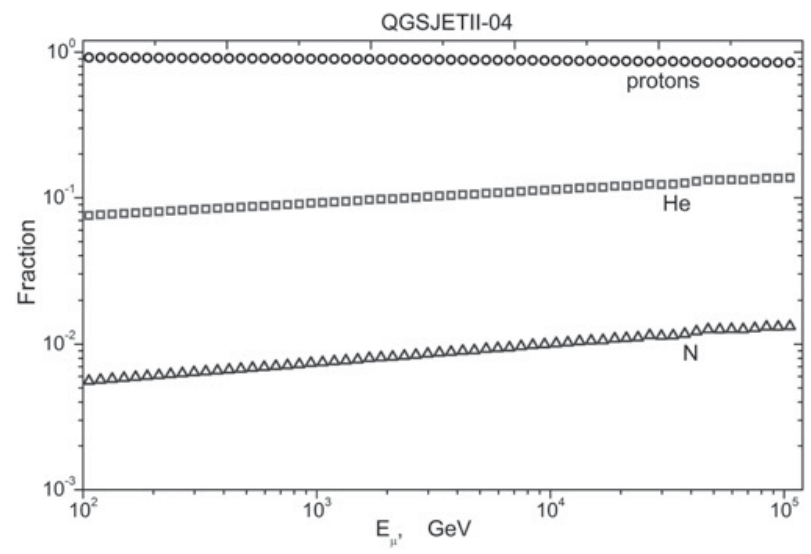

Figure 11. Relative contribution of protons, $\mathrm{He}$ and $\mathrm{N}$ nuclei into the muon spectrum.

coefficient 1.27 used by the TA collaboration [9] to decrease the energy estimates of showers calculated on the base of signals in the scintillation detectors may be understood as a result of this suppression. The increased intensity of the primary particle flux observed at the Yakutsk array at super high energies [31] may also be a result of smaller values of the calculated signals in surface scintillation detectors.
Authors thanks N. N. Kalmykov, pointing to alternative computing with other results and A. A. Lagutin for important assistance in the verification of the results for the QGSJET01 model.

\section{References}

[1] Kalmikov N. N., Ostapchenko S. S., Phys. Atom. Nucl. 56, 346 (1993)

[2] Ostapchenko S. S., Phys. Rev. D. 74, 014026; hep-ph/0505259 (2006)

[3] Ostapchenko S. S., Phys. Rev. D. 83, 014018 (2011)

[4] Ahn E.-J., Engel R., Gaisser T. K., et al., Phys. Rev. D. 80, 094003 (2009)

[5] Werner K., Liu F. M. and Pierog T., Phys. Rev. C. 74, 044902; hep-ph/0506232 (2006)

[6] Pierog T. and Werner K., Nucl. Phys. Proc. Suppl. 196, 102; arXiv:0905.1198 (2009)

[7] Ranft J., Phys. Rev. D. 51, 64 (1995) hep-ph/9911213 and hep-ph/9911232 (1999)

[8] Werner K., Phys. Rep. 232, 87 (1993)

[9] Abu-Zayyad T., Aida R., Allen M., et.al., (Telescope Array Collaboration) Astrophys. J. Lett. 768:L1 5 (2013)

[10] Pierog T., EPJ Web of Conf. 99, 09002 (2015)

[11] Ostaphcenko S. S., Progr. of Theor. Phys. Suppl., 193, 204 (2012)

[12] D’Enterria D., Engel R., Pierog T., et. al., Astropart. Phys. 35, 98 (2011)

[13] Engel R., Rebel H., Acta Phys. Polonica B. 35, 321 (2004)

[14] Achard P., et al. (The L3 Collab.) Phys. Lett. B. 598, 15-32, hep-ex/0408114v1 (2004)

[15] Ambrosio M., Antolini R., Auriemma G., et al., (The MACRO Collab.), Phys. Rev. D. 52, 3793 (1995)

[16] Aglietta M., Alpat B., Alieva E. D., et al., (The LVD Collab.), Phys. Rev. D. 58, 092005, hep-ex/9806001v1 (1998)

[17] Heck D., Knapp J., Capdevielle J.-N., et al., Report FZKA 6019 Forschungszentrum Karlsruhe, (1998)

[18] Panov A. D., Adams J. H., Ahn H. S. et al., Bull. Russ. Acad. Sci. Phys. 71, 494 (2007); Bull. Russ. Acad. Sci. Phys. 73, 564 (2009)

[19] Ahn H. S. (for the CREAM Collab.), Astrophys. J. Lett. L 89, 714 (2010)

[20] Derbina V. A., Galkin V. I. and Hareyama M. (for the RUNJOB Collab.), Astrophys. J. L 41, 628 (2005)

[21] Aguilar M., Aisa D., Alpat B., et. al., (AMS Collab.) Phys. Rev. Lett. 114, 171103 (2015)

[22] Aguilar M., Aisa D., Alpat B., et. al., (AMS Collab.) Phys. Rev. Lett. 115, 211101 (2015)

[23] Adriani O., Barbarino G. C., Bazilevskaya G. A., et. al., (PAMELA Collab.) Advances in Space Research 51, 219-226 (2013)

[24] Gaisser T. K. and Honda M., Ann. Rev. Nucl. Part. Sci. 52, 153-199 (2002) 
[25] Battistoni G., Ferrari A., Muraro S. and Sala P. R., (Proc. Suppl.) Nucl. Phys. B. 168, 286 (2007)

[26] Dedenko L. G., Roganova T. M., Fedorova G. F., JETP Lett. 100, 223 (2014)

[27] Dedenko L. G., Roganova T. M., Fedorova G. F., Phys. Atom. Nucl. 78, page numbers (2015)

[28] Dedenko L. G., Lukyashin A. V., Fedorova G. F. and Roganova T. M., EPJ Web of Conferences 99, 10003 (2015)
[29] Dedenko L.G., Zatsepin G.T., Proceedings of the 6th ICRC, Moscow, II, Extensive air showers and cascades process, 201-208 (1960)

[30] Lagutin A. A., Tyumentsev A. G., Yushkov A. V., J. Phys. G. 30, 573-596, hep-ph/0402070 Journal (2004)

[31] Glushkov A. V., Egorova V. P., Ivanov A. A., et al., Proceedings ot the 28th ICRC, Tsukuba 1, 393 (2003) 